Documentation et bibliothèques

DOCUMENTATION BIBLIOTHËQUES

\title{
Statistiques 2004-2005 et 2005-2006 des bibliothèques universitaires et de recherche au Canada
}

\section{My Loan Duong}

Volume 53, numéro 2, avril-juin 2007

URI : https://id.erudit.org/iderudit/1029238ar

DOI : https://doi.org/10.7202/1029238ar

Aller au sommaire du numéro

\section{Éditeur(s)}

Association pour l'avancement des sciences et des techniques de la documentation (ASTED)

\section{ISSN}

0315-2340 (imprimé)

2291-8949 (numérique)

Découvrir la revue

\section{Citer cet article}

Duong, M. L. (2007). Statistiques 2004-2005 et 2005-2006 des bibliothèques universitaires et de recherche au Canada. Documentation et bibliothèques, 53(2), 115-118. https://doi.org/10.7202/1029238ar

Tous droits réservés (C) Association pour l'avancement des sciences et des techniques de la documentation (ASTED), 2007
Ce document est protégé par la loi sur le droit d'auteur. L'utilisation des services d'Érudit (y compris la reproduction) est assujettie à sa politique d'utilisation que vous pouvez consulter en ligne.

https://apropos.erudit.org/fr/usagers/politique-dutilisation/ 


\title{
Statistiques 2004-2005 et 2005-2006 des bibliothèques universitaires et de recherche au Canada ${ }^{*}$
}

\author{
MY LOAN DUONG \\ my.loan.duong@umontreal.ca \\ Bibliothécaire à la Bibliothèque de bibliothéconomie et des sciences de l'information, \\ Université de Montréal
}

$\mathrm{D}$ EPUIS 1969, CHAQUE AUTOMNE, la parution des statistiques des 27 grandes bibliothèques des universités canadiennes, de l'Institut canadien de l'information scientifique et technique (ICIST), de Bibliothèque et Archives Canada (BAC) et de la Bibliothèque du parlement, regroupés dans l'Association des bibliothèques de recherche du Canada/Canadian Association of Research Libraries, est un événement attendu. Pour ceux et celles qui travaillent dans le milieu des bibliothèques d'université et de recherche, bibliothécaires et administrateurs qui en ont la charge, ces statistiques sont le baromètre de l'état de ces institutions et servent à faire le bilan des réalisations, à mesurer la progression de chacune et à définir les tendances pour orienter les actions futures.

Notons que les chiffres analysés ici, pour l'Université de Montréal (UdeM), sont les statistiques de l'UdeM seule, excluant les Hautes Études commerciales et l'École Polytechnique.

Pour des fins de comparaison avec les moyennes provinciales et nationale, les universités sont regroupées par régions: le premier groupe, dansl'Ouest, est constitué des universités de Colombie-Britannique, Simon Fraser et Victoria ; le deuxième groupe est composé des universités de l'Alberta, de Calgary, du Manitoba, de Regina et de la Saskatchewan ; l'Ontario, qui constitue le troisième groupe, comprend les universités Carleton, de Guelph, McMaster, d'Ottawa, Queen's, de Waterloo, Western Ontario, de Windsor et York. Viennent ensuite le quatrième, qui regroupe les universités du Québec dont Concordia, Laval, Montréal, et Université du Québec (UQ) et, enfin, le cinquième groupe, formé par les universités des Maritimes : Dalhousie, Memorial et du Nouveau-Brunswick.

Quatre indicateurs constituent les outils pour mesurer l'indice de qualité et de développement des bibliothèques universitaires et de recherche au Canada : "Dépenses et collections" (Expenditures and collection size), « Services émergents " (Emerging services), "Utilisation, installations et services" (Use, facilities and services) et «Salaires » (Salaries).

* «Chiffres et tendances » pour 2004-2005 et « Salaires » pour 2005-2006.

\section{Dépenses et collections}

En ce qui a trait aux collections et aux dépenses, on apprend que "l'investissement total dans les collections et les accès a été de 242,9 millions de dollars en comparaison à 236,1 millions de dollars pour l'année précédente ». La performance du dollar canadien, l'inflation générale contrôlée, les finances publiques plutôt favorables dans la plupart des provinces et la hausse du niveau de transfert aux universités ont permis aux administrateurs d'obtenir une augmentation annuelle de 2,9\%, légèrement supérieure à l'indice de $2,4 \%$ des prix à la consommation au Canada (p. $8^{1}$ ).

À l'ère de l'électronique, bâtir les collections, c'est aussi bâtir les réseaux pour y accéder. Ainsi, la migration de l'imprimé vers l'accès électronique se confirme aussi bien pour les périodiques que pour les monographies

\section{Périodiques}

Au total, on a dépensé davantage pour les périodiques électroniques que pour les imprimés en 2004-2005. Dans l'ensemble des bibliothèques, les achats de périodiques électroniques ont connu une hausse de $20,2 \%$, pour un total de 94,3 millions de dollars, tandis que les dépenses pour les périodiques imprimés ont diminué de $2,9 \%$, pour un montant total de 69,6 millions de dollars. En 2004-2005, les bibliothèques de l'ABRC détiennent " 290520 titres de périodiques en format imprimé ou en microformes et 451520 titres en format électronique ».

À titre indicatif, l'Université McGill est abonnée à 35597 périodiques en ligne et à 13836 en format imprimé et microformes ; l'Université de Toronto est abonnée à 28872 périodiques en ligne et à 32485 en format imprimé et microformes; à l'Université Laval, on compte 15640 périodiques en format électronique et 6612 en format imprimé et microformes. À l'UdeM, le nombre de périodiques en format électronique est de 13285 titres et de 8566 titres en format imprimé et microformes (Tableau IV : «Serial collections », p. 22 et 23).

1. Toutes les références renvoient au document suivant :

Statistiques de l'Association des bibliothèques de recherche du Canada/ABRC (Canadian Association of Research Libraries/CARL Statistics), 2004-2005, <www. carl-abrc.ca>. (Autre titre: CARL Statistics/Statistiques ABRC.) 


\section{Avec le développement des technologies de l'information et des communications, dans la plupart des bibliothèques de l'ABRC, des services nouveaux ou émergents de l'ère électronique font leur apparition.}

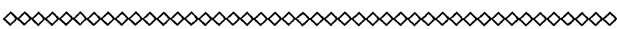

\section{Monographies}

Les dépenses totales pour les monographies, tous formats, ont augmenté de 4,5\% par rapport à l'année 2003-2004, pour un montant total de 59,3 millions de dollars. Les coûts d'accès aux monographies électroniques continuent d'augmenter. En 2004-2005, 24 bibliothèques de l'ABRC ont dépensé 4,81 millions pour l'accès aux monographies électroniques. L'UdeM a dépensé 44866 \$, McGill 329073 \$ (ce montant comprend la reconduction de l'accès aux monographies électroniques engagé en 2003-2004) et Toronto 679323 \$ (Tableau $\mathrm{V}$ : «Library materials expenditures », p. 24 et 25).

À l'Université de Toronto, qui compte la plus grosse collection parmi les bibliothèques universitaires (BU) canadiennes avec 10,3 millions de titres, les dépenses pour l'accès aux monographies électroniques ont plus que triplé : 32242 accès pour 143855 titres acquis au cours de l'année. L'UdeM compte aussi parmi les bibliothèques dont les achats de monographies en ligne sont les plus importants, avec 4650 titres sur un total de 33148 titres acquis en 2004-2005. À l'Université Laval, près de la moitié des monographies acquises, soit 15876 titres sur 32 600, sont en format électronique (Tableau III : «Monographs collections », p. 21).

En 2004-2005, les collections des 28 bibliothèques de l'ABRC se sont enrichies de 1,52 millions de volumes. $\mathrm{Au}$ total, les 29 bibliothèques de l'ABRC détiennent 81,8 millions de volumes, soit $1,9 \%$ d'augmentation par rapport à l'année précédente (p. 9).

\section{Élagage}

Qui dit développement des collections, dit aussi élagage. En effet, optimiser l'espace de conservation est une préoccupation constante des administrateurs de bibliothèques. Le problème de l'espace est un facteur important dans la décision de conserver ou non des ressources désuètes et peu utilisées. Il ne faut donc pas s'étonner que certaines bibliothèques, comme àl'UQAM par exemple, aient retiré plus de documents qu'elles n'en ont ajouté à leurs collections. Au total, dans l'ensemble des bibliothèques de l'ABRC, 400 ooo titres ont été retirés des collections en 2004-2005. L'UdeM a retiré 20623 volumes de ses collections (Tableau I : "Library collections", p. 16 et 17 ).
Toutes les bibliothèques universitaires sont subventionnées par des fonds gouvernementaux, mais les grandes bibliothèques peuvent compter aussi sur le financement externe qui, dans quelques cas, fait parfois toute la différence. À la colonne "external fundings", on constate que les subventions de sources autres que gouvernementales varient beaucoup d'une bibliothèque à l'autre. Tous les chiffres ne sont pas disponibles, mais McGill et Concordia, par exemple, ont pu compter sur d'importantes subventions de sources extérieures (Tableau V : «Library materials expenditures », p. 25).

$\mathrm{Au}$ chapitre des dépenses, une intéressante statistique concerne le pourcentage que chaque bibliothèque consacre aux ressources, aux salaires et aux frais d'opération. Par exemple, l'UdeM consacre 30,54 \% des dépenses aux ressources, $64,94 \%$ aux salaires et $4,51 \%$ aux frais d'opération. En comparaison, McGill consacre $34,60 \%$ aux ressources, $61,20 \%$ aux salaires et $4,18 \%$ aux frais d'opération, tandis que Toronto consacre $46,97 \%$ de son budget aux ressources, $48,26 \%$ aux salaires et $4,75 \%$ aux autres frais (Tableau VII : «Summary of library expenditures", p. 28).

Les dépenses consacrées aux ressources constituent l'indicateur de performance par excellence. Sans surprise, c'est l'Université de Toronto qui mène le bal avec 9339649 \$ pour les monographies et 14982960 \$ pour les périodiques, suivie de l'Université d'Alberta qui a investi $5368076 \$$ dans ses monographies et $9794761 \$$ dans ses périodiques. McGill fait bonne figure avec 3518145 \$ pour les monographies et 8145878 \$ pour les périodiques. À Laval, les dépenses pour les monographies se sont élevées à $2066285 \$$ et à $7533055 \$$ pour les périodiques. L'UdeM a dépensé $2398406 \$$ pour enrichir sa collection de monographies et 6884901 \$ pour les périodiques (Tableau V : «Library materials expenditure ", p. 24 et 25).

\section{Les services émergents}

Le renouvellement des infrastructures technologiques a été une préoccupation importante des bibliothécaires et des administrateurs depuis la fin des années 1990 et le début des années 2000.

Avec le développement des technologies de l'information et des communications (TIC), dans la plupart des bibliothèques de l'ABRC, des services nouveaux ou émergents de l'ère électronique font leur apparition. On assiste à la mise en place des carrefours d'information (Information Commons) qui offrent des aménagements physiques favorables à la concertation, la recherche et l'exploitation des ressources. Selon les institutions, ces services sont administrés soit par la bibliothèque, soit par la bibliothèque avec un des départements de l'université. Dans plusieurs bibliothèques universitaires anglophones (McGill, Alberta, Calgary, Toronto, p. 11), ils sont subventionnés par le budget ordinaire ou par des fonds spéciaux. L'aide à l'édition électronique des travaux et des thèses, la publication et l'accès en ligne 
de la production des chercheurs et des professeurs, et les projets de numérisation font partie aussi des services émergents dans les bibliothèques universitaires et de recherche de même que la référence en ligne, la vidéoconférence, etc.

Certaines universités mettent à la disposition des usagers une panoplie d'outils informatiques pour la consultation des ressources, dont les ordinateurs portables. L'accès à un réseau sans fil est disponible dans la plupart des bibliothèques.

\section{L'utilisation, l'installation et les services}

$\mathrm{La}$ formation des usagers, au cours des dernières années, a été une préoccupation majeure des bibliothécaires. Dans plusieurs universités canadiennes, à l'instar de nos voisines du Sud, des cours crédités sont offerts pour la recherche et l'exploitation des ressources documentaires. Le présent rapport n'en fait cependant pas mention. Les statistiques de l'ABRC portent plutôt sur le nombre de présentations de la bibliothèque à des groupes ("Numbers of library presentations to groups"). $\grave{A}$ ce chapitre, il est intéressant de noter que les bibliothécaires de l'UdeM sont parmi ceux qui offrent le plus grand nombre de présentations de groupe, si l'on inclut dans ces présentations les visites des bibliothèques et les formations à la recherche des ressources documentaires (Tableau I : "Collections uses and facilities", colonne "Number of library presentations to groups", p. 46 et 47 ).

\section{L'indice de l'utilisation des ressources : constat troublant}

Le principal indicateur de l'utilisation des services étant la circulation des documents, un constat troublant se dégage. On note une diminution de $8,3 \%$ du nombre des prêts dans $21 \mathrm{BU}$ par rapport à l'année précédente, avec 11,9 millions en 2004-2005 et ce, malgré une augmentation des inscriptions d'étudiants. Et même si le volume des prêts initiaux a crû de $5 \%$ par rapport à l'année antérieure, il reste plus faible que l'augmentation des inscriptions d'étudiants. L'utilisation interne est aussi en baisse dans 19 bibliothèques et la tendance est aussi à la baisse dans le volume de demandes de PEB et dans les chiffres des livraisons. Pour 26 des bibliothèques (sauf celle de l'Université de l'Alberta), les prêts sont passés de 549000 en 2002-2003 à 494000 en 2003-2004 et 488 ooo en 2004-2005. Pourtant, il est évident qu'aucune bibliothèque de recherche ne peut combler tous les besoins de ses usagers à partir des seules ressources disponibles sur place.

Notons que les statistiques ne font pas état de la consultation des ressources électroniques, comme les bases de données en ligne ou des documents déposés à la réserve électronique. Cette lacune dans la compilation des statistiques serait-elle une des raison de la dimi-
C'est au Québec que le salaire moyen, avec 67758 \$, et le salaire médian, avec 67199 \$, sont les plus bas pour les bibliothécaires professionnels.

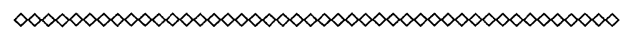

nution constatée au niveau de la consultation et du prêt des ressources?

Rares sont les bibliothèques, comme celle de l'Université de Waterloo, qui offrent l'accès électronique aux documents de la réserve (p. 51, $\mathrm{n}^{\circ} 18$, «Endnotes to use, facilities and services questionnaire ").

Ce sont deux universités montréalaises, l'UdeM et McGill, qui montrent le niveau de fréquentation le plus élevé : 2386215 usagers ont franchi les tourniquets des bibliothèques de l'UdeM et 3216504 , ceux de l'Université McGill en 2004-2005 (Tableau I : «Collection uses and facilities », p. 47).

\section{Les personnels et les salaires}

On a beaucoup parlé de départs massifs, mais il semble que le nombre de professionnels ait peu augmenté dans les BU en 2004-2005, sauf à Bibliothèque et Archives du Canada (BAC) en raison de la fusion de l'ancienne Bibliothèque nationale du Canada et des Archives nationales du Canada (p. 11). C'est l'UdeM qui détient le record du nombre d'employés non cadres, avec 322 personnes, et en nombre de bibliothécaires cadres (30 cadres pour 54 professionnels).

\section{La disparité des salaires entre les bibliothécaires}

Le tableau "Salary data for all professionals" (p. 54), qui recense les données sur le salaire moyen et le salaire médian de tous les professionnels regroupés par province indique que c'est au Québec que le salaire moyen, avec 67758 \$, et le salaire médian, avec 67199 \$, sont les plus bas pour les bibliothécaires professionnels. Les salaires médian et moyen des bibliothécaires de l'UdeM sont les plus bas de la province, avec 63214 \$ et $64116 \$$. Notons que le salaire médian des bibliothécaires professionnels, au niveau national, se situe à 72874 \$ et le salaire moyen, à 72868 \$.

Le tableau "Salary data for administrative librarians" (p. 55) qui recense le revenu des bibliothécaires cadres au Québec montre un salaire moyen de 81967 \$ et un salaire médian de $80440 \$$. Ces salaires des bibliothécaires-administrateurs québécois semblent concurrentiels par rapport à ceux de leurs collègues des autres provinces, compte tenu du coût de la vie au Québec. C'est dans la province de l'Ontario que le salaire moyen des bibliothécaires cadres est le plus élevé (89 266 \$), tandis 


\section{Le déclin continu dans les indicateurs d'utilisation des bibliothèques, qui ne peut être attribué totalement à la révolution électronique, est une source de préoccupation.}

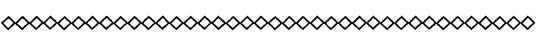

que ce sont les bibliothécaires-admistrateurs de l'Ouest qui ont le salaire médian le plus élevé, avec 90991 \$. C'est à l'UdeM qu'on trouve le plus grand nombre de cadres avec 30 bibliothécaires-administrateurs. Dans les autres universités québécoises, on compte 15 bibliothécaires occupant des fonctions administratives à McGill, 14 à Laval, 7 à Québec, 6 à Sherbrooke et 10 à Concordia.

Le tableau "Salary data for non-administrative librarians" (p. 56) confirme les données du tableau "Salary data for all professionals" (p. 54), en ce qui concerne le salaire moyen et le salaire médian des bibliothécaires occupant des postes non administratifs à l'UdeM. On voit que le salaire médian des bibliothécaires de l'UdeM est parmi les plus bas de toutes les provinces avec 63214 \$, ceux des autres universités québécoises étant de 67032 \$ à McGill, de 68283 \$ à Laval, de 68434 \$ à Concordia et de 64078 \$ à Sherbrooke.

Les bibliothécaires de l'Université Dalhousie gagnent en moyenne 55871 \$ et ceux de l'Université du Nouveau-Brunswick, $59652 \$$. À Toronto, le salaire médian est de $70323 \$$. Le salaire médian national est de $67519 \$$.

Notons que l'Institut de la statistique du Québec a confirmé le retard des employés non cadres des secteurs public et parapublic québécois par rapport à leurs collègues occupant des fonctions équivalentes dans le secteur public québécois : "En 2006, dans les emplois repères étudiés, le salaire des employés de l'administration québécoise est en retard de 15,2\% par rapport à celui de l'ensemble des autres salariés québécois travaillant dans les établissements de 200 employés et plus. Un retard est aussi noté vis-à-vis du secteur privé (-15,7\%). " Ces propos sont tirés de deux communiqués émis par le gouvernement du Québec le 30 novembre $2006^{2}$.

De toute évidence, plus les travailleurs d'une organisation ont de l'expérience, plus sa masse salariale est importante. Les années d'expérience au travail, outre le fait qu'elles constituent une source de richesse, ont de même un impact important sur la masse salariale d'une institution. À l'UdeM, par exemple, en raison l'embauche d'un grand nombre de nouveaux employés cadres et professionnels, la moyenne des années d'ex-

2. Fiche méthodologique : Rapport rémunération des salariés - État et évolution comparés - La comparaison de la rémunération globale des salariés (Partie I) Rémunération globale des salariés de l'administration québécoise : retard face aux autres salariés québécois œuvrant dans les établissements de 200 employés et plus. http://communiques.gouv.qc.ca/cgi-bin/search.pl périence des bibliothécaires (14,78 années) est un peu inférieure à la moyenne canadienne ( 16,14 années) et à la moyenne provinciale (15,04 années).

Le tableau «Salary distribution 2005-2006" (p. 58) est fort intéressant car il confirme le fait que les bibliothécaires des universités francophones, sauf pour le personnel administratif, ont un salaire de beaucoup inférieur à celui de leurs collègues anglophones. L'examen du tableau montre également quau Québec, l'UdeM compte 8 bibliothécaires gestionnaires qui touchent un salaire de 80 ooo $\$$ et plus et 17 bibliothécaires administratifs, un salaire de 65000 et plus. Dans l'ensemble du pays, c'est à l'Université de Montréal que l'on trouve le plus grand nombre de bibliothécaires $-47-$, dans la catégorie des salaires de 52000 \$ à $64000 \$$. McGill, Concordia, de même que les universités de Toronto et de l'Alberta se démarquent avec une forte proportion de bibliothécaires touchant $65000 \$$ et plus.

Le tableau "Salary distribution: Average and median salary per year of professional experience Librarian position only 2005-2006" (p.60 et 61) indique qu'à l'UdeM, le salaire des bibliothécaires qui ont moins de 7 ans d'expérience est de 52646 \$, plus élevé que dans la plupart des bibliothèques québécoises, sauf à l'Université Sherbrooke ( 54769 \$). La moyenne au Québec est de 49295 \$, la moyenne nationale est de 54309 \$.

Qu'on le veuille ou non, dans tout classement, il y a une compétition implicite. Il y a certainement une disparité sur le plan salarial dans les universités francophones. À d'autres niveaux, dans le groupe des G1o, que ce soit en matière de collections, ou au chapitre des sommes investies dans les collections, des subventions directes ou indirectes, des infrastructures et des salaires, les BU anglophones ont une avance sur les BU francophones. Néanmoins, il y a eu des progrès réalisés au cours des dernières années dans les BU francophones, notamment à l'UdeM où beaucoup d'efforts ont été faits sur le plan des infrastructures et du développement des collections.

Quel est le constat de ce portrait des bibliothèques universitaires au Canada et au Québec en 2004-2005?

En conclusion du rapport, comme l'affirme David Holmes, ancien directeur de la recherche et de la planification institutionnelles à l'Université Carleton, qui a rédigé la note introductive de cette livraison, «l'année 2004-2005 a été une bonne année pour les bibliothèques de $l ' A B R C$ " grâce à plusieurs facteurs conjugués, dont une " amélioration des finances publiques dans l'ensemble $d u$ pays et une augmentation du niveau de transferts aux universités ». Par contre, David Holmes souligne que "le déclin continu dans les indicateurs d'utilisation des bibliothèques, qui ne peut être attribué totalement à la révolution électronique, est une source de préoccupation qu'il faudrait examiner ». (-) 